Biological and Clinical Sciences Research Journal

ISSN: 2708-2261

www.bcsrj.com

DOI: https://doi.org/10.54112/bcsri.v2021i1.69

Biol. Clin. Sci. Res. J., Volume, 2020: 69

Review Article

\title{
ROLE OF CRISPR TO IMPROVE ABIOTIC STRESS TOLERANCE IN CROP PLANTS
}

FAROOQ MU, *BASHIR MF, KHAN MUS, IQBAL B, ALI Q

\author{
Institute of Molecular Biology and Biotechnology, The University of Lahore, Lahore, Pakistan \\ Corresponding author email: chfarhan821@ gmail.com
}

(Received, $26^{\text {th }}$ January 2021, Revised $12^{\text {th }}$ May 2021, Published $19^{\text {th }}$ May 2021)

\begin{abstract}
The study for genetic variation in plant genomes for a variety of crops, as well as developments of genome editing techniques, have made it possible to cultivate for about any desired trait. Zinc finger enzymes; have made strides in genome-editing. Molecular biologists can now more specifically target every gene using transcription activator-like effector nucleases and ZFNs. These methods, on the other hand, are expensive and timeconsuming because they involve complex procedures. Referring to various genome editing techniques, CRISPR/Cas 9 genetic modification is simple to construct and clone and the Cas 9 could be used with different guide RNAs controlling different genes. Following solid evidence demonstrations using the main CRISPR-Cas9 unit in field crops, multiple updated Cas 9 cassettes are often used in plant species to improve target precision and reduce off target cleavage. Nmcas 9 , Sacas 9 , as well as Stcas 9 are a few examples. Furthermore, Cas 9 enzymes are readily available from a variety of sources. Bacteria that had never been discovered before has found solutions available to improve specificity and efficacy of gene editing techniques. The choices are summarized in this analysis to plant's experiment to develop crops using CRISPR/Cas9 technology; the tolerance of biotic \& abiotic stress may be improved. These strategies will lead to the growth of non-genetically engineered crops with the target phenotype, which will further improve yield capacity under biotic \& abiotic stress environments.
\end{abstract}

Keywords: CRISPR, Crops, Quantitative trait loci, biotic stress, TALEN, abiotic stress, ZFN

\section{Introduction}

In the present situation, the most critical problem facing humanity is ensuring food sustainability for an expanding world population. By 2050, the global population may have increased to ten billion people, necessitating a 60-100percent annual rise in global food demand. Extreme weather, limited agricultural land supply, and rising biotic \& abiotic pressures, in addition to the raising population rate, are important constraints for food production. The development of technology that can aid in crop enhancement can help to boost output to some point. Physical, biochemical, \& biological (T-DNA transposons) metagenomic methods have made significant contributions in understanding the function of genes \& transposons. For the determination the biological processes that have been resulted from the improved crop populations during recent decades (Ma et al., 2016); the transgenic technologies have been used to learn about basic plant science as well as to improve crops for the past 3 decades. After all, whenever it relates to food crop plants, the implementation of transgenic organisms into target genes are non-specific, occasionally unstable, as well as a source of public consideration (Shimatani et al., 2017). Any use of genome editing technology of sequence-specific nucleases (SSNs) has shown accurate gene editing for both animal \& plant environments over the last decades. Double stranded breaks in the target genes are caused by these SSNs. The Non-homologous end joining (NHEJ) and homology directed rearrangement pathways are used to repair doublestrand breaks (DSBs), resulting in insertion or deletion and replacement mutations throughout the target region (Jinek et al., 2012). Genome editing techniques, in comparison to transgenic approaches, which result in spontaneous implantations or in many cases, random phenotypes, yield identified mutations, making them a useful tool in practical genomics as well as crop breeding. Genome modified crops have such a benefit over transgenic crops in that they 'carry' the target trait's edited DNA (Mali et al., 2013). When opposed to traditional genetically modified plants, such improved plants can also be used in development programs, as well as the resulting plants can be used immediately with fewer acceptability or consumption problems and fewer regulatory procedures (Waltz, 2018). The benefits and uses of $2^{\text {nd }}$ generation genetic modification technologies like CRISPR/Cas9 as well as its variants over $1^{\text {st }}$ generation genetic modification methods like meganucleases, ZFNs and transcription activator-like effectors nucleases. 


\section{Genome editing throughout the new period of engineered enzymes}

A pattern of DNA binding domains has been fused to a non-specific enzyme domain in genetic engineering. The intended gene can also be precisely cleaved by such fused nucleases, as well as the damage can be restored using NHEJ and Homology directed repair (HDR), thus the name "genome editing" (Gaj et al., 2013). The genetic engineering techniques such as meganucleases and Transcription activator-like effector nucleases (TALENs) require labour intensive as well as time-consuming techniques to achieve goal precision. Secondgeneration genetic modification methods, such as CRISPR or Cas9, necessitate simpler design and implementation methodologies, as well as being less expensive and time consuming. For even more than a decade, ZFNs have been extensively used for genetic modification in both animal \& plant systems (Govindan and Ramalingam, 2016). Due to poor target accuracy, labour intensive nature, numerous off target cleavages, and a small number of possible active sites, ZFNs have been now less favored (Chen and Gao, 2013; Kim et al., 2018; Osakabe, 2015). TALENs are created by altering activator effector domain sequences for desired target tracking, then fusing them with the FokI enzyme to create a TALEN that can be used to change target genomes. Engineered TALENs, like ZFNs, recognize 18-20 base pairs sequences, with a $14-20$ base pair spacer required for FokI dimerization (Ueta et al., 2017). Because of their duration, TALENs have a higher average target affinity than ZFNs. TALENs, on the other hand, are difficult to plan and assemble due to the necessity of a $\mathrm{T}$ base only at the starting point, as well as their large scale and repetitiveness like in tobacco and brachypodium (Zhang et al., 2013: Shan et al., 2013). TALENs were used to modify the genomes of plants such as Arabidopsis thaliana (Cermak et al., 2011), rice (Li et al., 2015).

\section{CRISPR/Cas9}

The CRISPR/Cas9 genetic modification method was first described in mammals in 2012, as well as its effectiveness in genetic modification has changed the world studies in plants and animals biology (Jinek et al., 2012). Unlike ZFNs \& TALENs, CRISPR genetic engineering is simpler, requiring the development of a reference RNA of around 20 nucleotides that is comparable to target nucleotides in DNA stretch. CRISPR stands for "Clustered Regularly Interspaced Short Palindromic Repeats (Jansen et al., 2002). Relates to Random copies flanked with - anti DNA sequences which were first discovered in the Escherichia coli Intracisternal A particles (iap) genomes' downstream regions (Ishino et al., 1987). External DNA sequences originating from plasmid DNA \& phages were shown to be structurally similar to any of these - anti sequencing. Following that, the process of template cleavage was investigated for genetic modification, as well as CRISPR/Cas9 cleavage new technologies 'arrived' as just a successful genome editing method (Malony et al., 2005; Li et al., 2018).

The CRISPR cleaved process requires a synthetic RNA template of 20 nucleotides that binds to the DNA Template and (ii) the RNA-guided nuclease enzyme, which cleaves three or four bases just after the nucleotide binding motif (Jinek et al., 2012). Since its inception, the CRISPR cleavage technique has been widely used in genome editing in a variety of species. Between 2010 well as 2018, approximately 5000 papers documenting the use of CRISPR were written. The measures for executing a CRISPR program are as follows: (i) identifying the PAM segment in the host genome, (ii) analyzing a single gRNA, (iii) copying the sgRNA it into compatible binary vector, (iv) insertion into host species or cell lines transformations, (v) sampling, and (vi) testing of edited segments. Even a limited lab with such a basic genetic manipulation set up will carry out genetic modification trials due to the easy steps used in CRISPR/Cas9 facilitated genome editing. CRISPR/Cas9 methods were used to alter plant sequences more widely in recent years. ZFNs or TALENs are really a reflection of ZFNs or TALENs. It is easy to use. After all, in plants, the majority of the editing has already taken place. Arabidopsis, wheat, and other model species have all shown this. Only tobacco or a few crop varieties have been studied using CRISPR technologies (Jiang et al., 2013). The development of substantial off-target cleaved areas as a result of complex formation of a gRNA to misaligned comparable target DNA inside the gene is among the large shortcomings of the CRISPR/Cas9 technique. As a result, so many Cas9 enzymatic modifications have also been intended to enhance selectivity and decrease off target cleavage. A further strategy for reducing off-target cleavages is to extend the size of the nucleotide binding motif. Cas9 enzymes from various bacterial species would have unique as well as enlarged protospacer adjacent motif (PAM) segments, which could help increase on-target selectivity. The Nmecas9 CRISPR system, which was extracted from $N$. meningitidis, identifies an 8-mer PAM pattern (fifty-NNNNGATT), which can enhance target specificity as well as start reducing off target cleavage (Lee et al., 2016). 2 Cas9 casettes (st1cas9 and st2cas9) were collected from S. thermophilus. PRKDC as well as CARD11 are 2 human loci for which the st3cas9 was used to

[Citation: Farooq, M.U., Bashir, M.F., Khan, M.U.S, Iqbal, B., Ali, Q. (2021). Role of CRISPR to improve abiotic stress tolerance in crop plants. Biol. Clin. Sci. Res. J., 2021: 69. doi: https://doi.org/10.54112/bcsrj.v2021i1.69] 
edit. Compared to the prior years, there were fewer off-target levels for which SpCas9 was established (Pauwels et al., 2016; Hu et al., 2018) and used 50NGA PAM to effectively edit the genome. Finally, to increase target accuracy, multiple CRISPR/Cas9 homologues have been discovered (Zetsche et al., 2017). CpfI, unlike Cas9, cleaves with such a single RNA directed (crRNA) complex or creates cohesive endings with 4-5 nucleotide fifty-overhangs. The CRISPR/CpfI technique has been extensively used for both animals and plant systems, with few to no off--target effects. In addition to Cpf1, approximately 53 certain CRISPR/Cas9 targets have also been identified, including the $\mathrm{C} 2 \mathrm{c} 2$ enzyme by Leptotrichia shahii. It has dual enzyme activity and therefore can target single-strand RNA (Zhang et al., 2017).

\section{CRISPR for genetic modification in crops}

CRISPR has played an important role for genetic modification in crops through using Cas9 as well as gRNA is expressed in plant systems using plantspecific reverse transcriptase 3 promoters [AtU6 (Arabidopsis); TaU6 (wheat); OsU6 and OsU3 (rice). Add gene is a non-profit, global recombinant registry that can currently provide more than thirty empty gRNA Back-bones in binary vector 2 . The plant RNA polymerase III promoters as well as gRNA scaffold on the hollow gRNA back-bones can be used to implant the gRNA of importance.

\section{Use of CRISPR to improve crop production}

So far, the CRISPR or Cas9 gene editing system has been used in approximately twenty crop species (Ricroch et al., 2017) for a variety of traits such as yield enhancement and biotic as well as abiotic stress control. Several of the published papers are called solid evidence experiments since they explain the use of the CRISPR/Cas9 method to take out particular genes involved in abiotic as well as biotic stress tolerance mechanisms. Microbial communities cause biotic stress, which poses serious challenges throughout the growth of disease-resistant plants, accounting for more than $60 \%$ of possible yield reductions and $70-75 \%$ of global food output decreases (Ran et al., 2015; Ren et al., 2016). Crop stress resistance and immunity to large abiotic stresses including drought conditions have also been improved using CRISPR/Cas9-based genetic modifications. The following is a summary and use of CRISPR for genetic modification in multiple crop species.

Monocots (Rice)
Rice (Oryza sativa L.) is an important staple food crop for even more than 50 percent of the world's population. It is well-known among the global population and, owing to its limited genome size, it is well-known among scientists. Monocots have been analyzed and used as a sample crop. Several experiments have recently demonstrated the use of CRISPR-based genetic modification in rice, and a few experiments have documented the use of genome editing to increase herbicide resistance for rice crops production. The rice gene has a lot of possible PAM locations (one in ten base pair) (Osakabe and Osakabe, 2015; Xie and Yang, 2013). In the coming years, CRISPR technologies can also be used to explore any feature of concern in the genome sequence (Shan et al., 2013) demonstrated sequencespecific. For first time in every crops cell, CRISPR/Cas9 induced genomic alteration of 3 rice proteins, (OsPDS), and (OsBADH2), or mitogenactivated related protein (OsMPK2), and is used to monitor response to different abiotic stress signals also using protoplast \& particle deluged rice calli structures. For OsPDS and OsBADH2, edit levels of approximately $9 \%$ and $7 \%$, accordingly, were found (Xie and Yang, 2013). There have been developed 2 rice-specific genetic modification vectors, pRGE3 and pRGE6, to illustrate an RNA-guided genetic engineering method. Rice explants were used to assess a negative controller of biotic as well as abiotic stresses that was chosen for genetic manipulation using 3 gRNAs. A much more detailed gRNA architecture methodology led to a low level of the off target.

For a set of genes, include OsDERF1, OsEPSPS, and OsMYB5, the productivity of the CRISPR/Cas9 mechanism in causing targeted mutations and heredity in mutated rice varieties was assessed (Zhang et al., 2014). ). A broad range of mutation frequencies (21-66\%) was observed in $\mathrm{M}_{0}$ generation different genes of no or one base pairs off-target mutations. In the $T_{2}$ generation, homozygosity mutants accounted for up to $11 \%$ of all mutations. This activation induced nucleoside deaminase target method allowed for targeted bp editing of a herbicidal gene C287 of rice (Shimatani et al., 2017). Base edit without the use for DSBs was achieved using dCas9 fusion with $\mathrm{C}$ deaminase (Zong et al., 2017). Li et al. (2017) used the BE3 bases editor for base edit the rice OsPDS \& OsSBEIIb genes. $\mathrm{BE} 3$ editor is a new genetic modification technology that mixes nicked and unnicked DNA (Table 1).

[Citation: Farooq, M.U., Bashir, M.F., Khan, M.U.S, Iqbal, B., Ali, Q. (2021). Role of CRISPR to improve abiotic stress tolerance in crop plants. Biol. Clin. Sci. Res. J., 2021: 69. doi: https://doi.org/10.54112/bcsrj.v2021i1.69] 

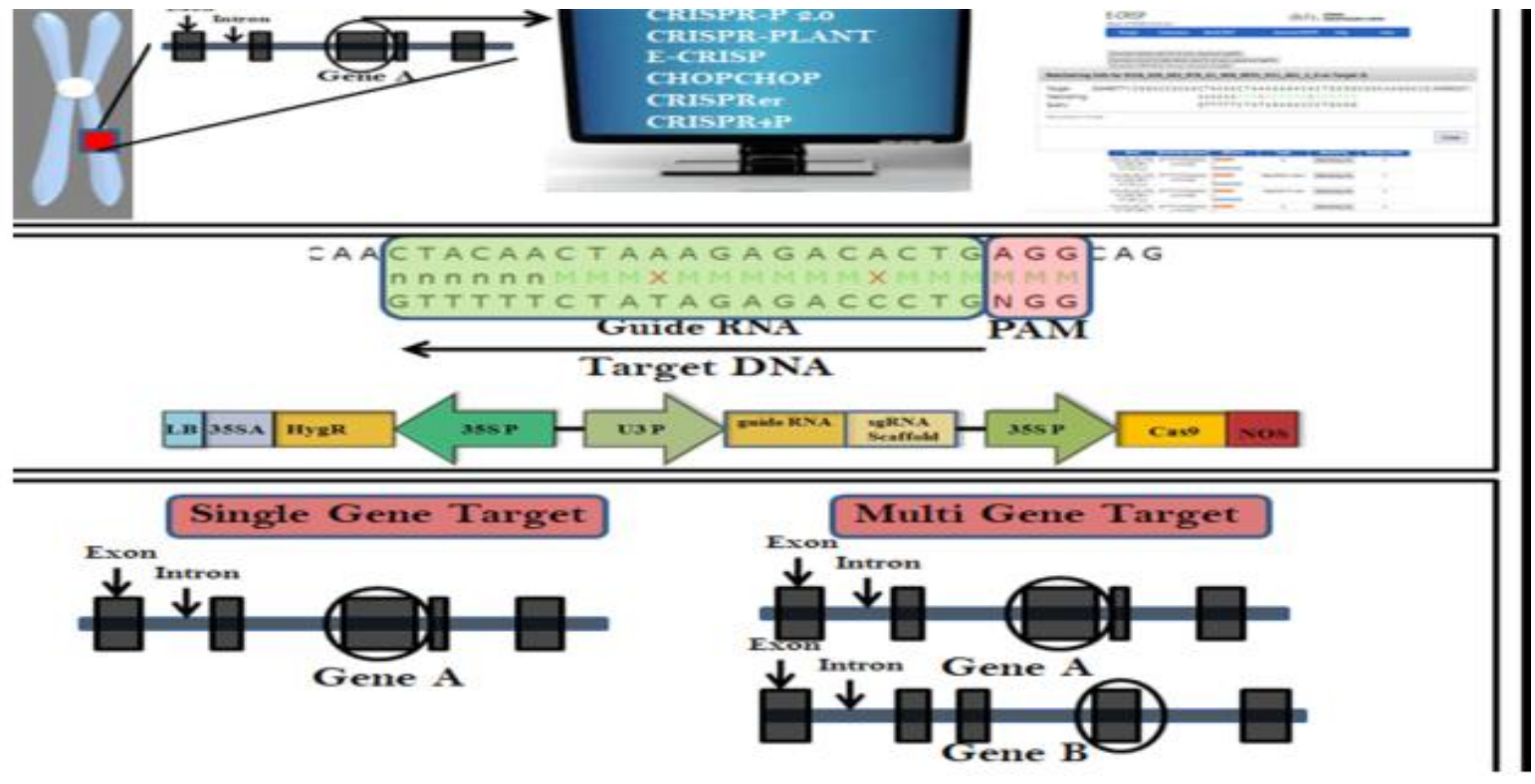

Figure 1 | Since 2005 through 2018, analysis \& review papers on TALEN, \& CRISPR were written. (A) The search terms ZFN, TALEN, or CRISPR were used also are using web of scientific web page throughout the title. Information was analyzed over two different time periods: 2010-2014, and 2015-2018.

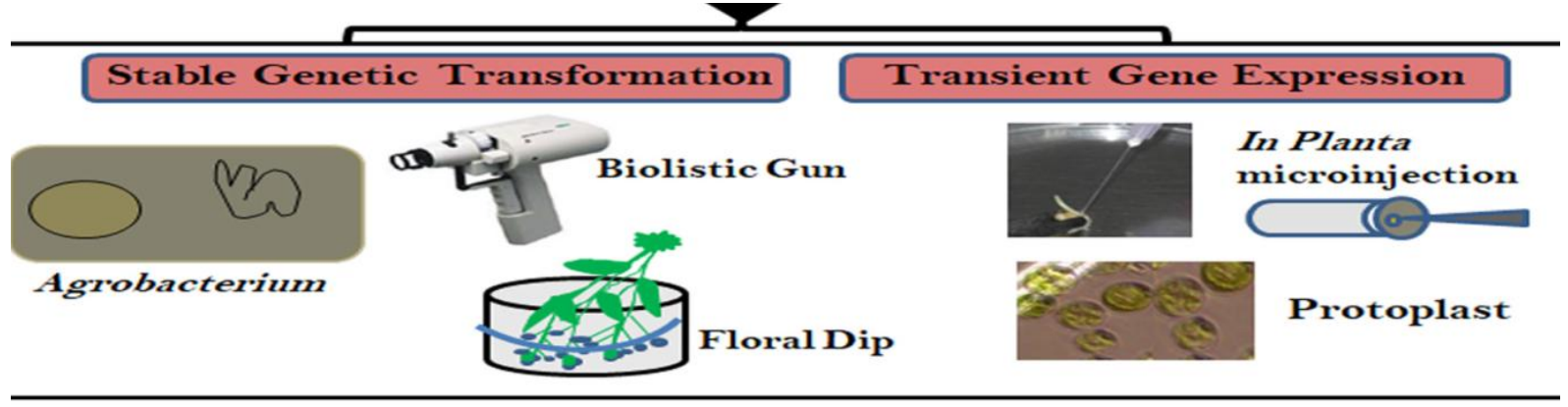

Restriction Enzyme

Sanger Sequencing/ NGS
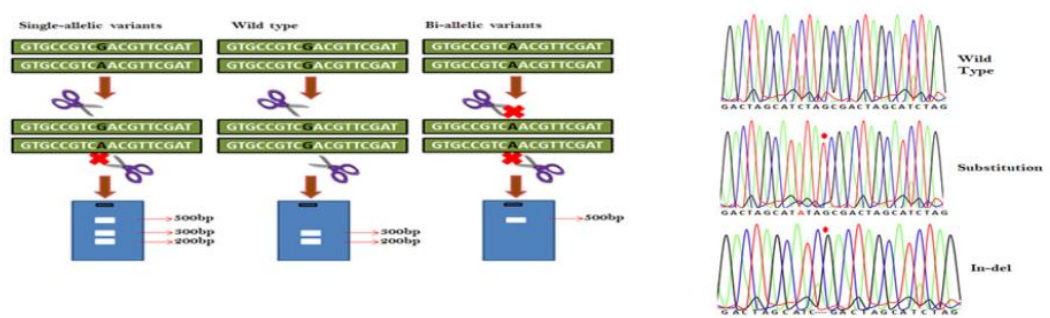

Real Time PCR

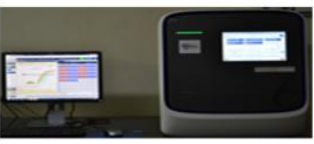

Figure 2 A flow map depicting the processes required in genome editing using CRISPR/Cas9. Initial step covers gene sorting \& gRNA design, while Step 2 covers cloning that gRNA into a binary vector. Step 3 displays the single \& multiplex editing options. Step 4 illustrates trans-formation method, Step 5 describes edited crops screening techniques, and Step 6 shows how to evaluate and pick the desired transgene plant for both the target trait. The efficient application of base pairs edit in rice was illustrated in this research. CRISPR/Cas9 allows for multiplex genetic modification with an almost infinite specific gene (Yang et al., 2017). ) and was shown in rice and Arabidopsis recently (Zhang et al., 2018). For each plant modification in rice, single binary vector was used to

[Citation: Farooq, M.U., Bashir, M.F., Khan, M.U.S, Iqbal, B., Ali, Q. (2021). Role of CRISPR to improve abiotic stress tolerance in crop plants. Biol. Clin. Sci. Res. J., 2021: 69. doi: https://doi.org/10.54112/bcsrj.v2021i1.69] 
accurately edit 8 agronomic genomes. The proteins were ligated with intermediary vectors using isocaudamer procedure. The study found that a cascade of RNAs had no effect on the genetic variation of CRISPR/Cas9, and that a cascade of sgRNAs had no effect on the mutation of CRISPR/Cas9 (Xu et al., 2015; Watanabe et al., 2018).

\section{Wheat}

Wheat is an important cereal grain; grown as a staple food crop all over the world (Shan et al., 2014). The CRISPR/TaMLO deletion has also been shown to impart tolerance to Blumeria graminis. Tritici downy mildew disorder (Btg).4 of the 7.2 $\mathrm{T}_{0}$ knock MLO grain homoeolog transgenic lines tested for restriction enzyme utilizing $\mathrm{T} 7$ endonuclease was found to have the restriction enzyme sequence edited (Wang et al., 2014). The numbers of transgenic lines produced can be improved or increased using build distribution methods. SSNs \& gRNA are usually delivered using T-DNA-based guidance systems. However, genome amplicons tend to improve gene targeting efficiency (Gil-Humanes et al., 2017) where have used grain dwarf-virus (WDV)-based Genome replicons of transient or straightforward production of Cas9 cassettes, culminating in a twelve-fold rise in ancestral ubiquitin expression of genes in hexaploid wheat. In the future, elevated gene targeting with WDV-based Genome replicons may be a viable approach for complex genome editing (Kim et al., 2018).

The CRISPR/Cas9 genetic modification method in wheat explants to edit two biotic stress genes, wheat

TABLE 1 Use of CRISPR-based genetic modification in plants to improve biotic, abiotic, \& nutritional traits

\begin{tabular}{|c|c|c|}
\hline Crop Plants & Target Gene & References \\
\hline Rice & $\begin{array}{l}\text { OsERF922 (ethylene-responsive } \\
\text { factor) }\end{array}$ & Wang et al., 2016 \\
\hline Rice (IR24) & OsSWEET13 & Zhang et al., 2017 \\
\hline Tomato & SIMAPK3 & Wang et al., 2015 \\
\hline Bread wheat & $\begin{array}{l}\text { TaMLO-A1, TaMLO-B1, } \\
\text { andTaMLOD1 }\end{array}$ & Wang et al., 2014 \\
\hline Tomato & Rin & Ito et al., 2015 \\
\hline Maize & ARGOS8 & Shi et al., 2017 \\
\hline Soybean & GmPDS11 \& GmPDS18 & Du et al., 2016 \\
\hline Potato & ALS1 & Butler et al., 2016 \\
\hline Wheat & TaVIT2 & $\begin{array}{l}\text { Cai et al., } 2018 \\
\text { nefits by Agrobacterium-mediated }\end{array}$ \\
\hline \multicolumn{2}{|c|}{$\begin{array}{l}\text { Maize } \\
\text { Maize is an important cereal grain, with phytic acid } \\
\text { accounting for more than a quarter of the plant. It is } \\
\text { thought to be nutritional and an environmental } \\
\text { contaminant since it is not absorbed by animals } \\
\text { (Liang et al., 2014). In Z. mays, researchers have } \\
\text { confirmed knocking out genes associated with phytic } \\
\text { acid synthesiss (ZmIPK1A and ZmMRP4) (Endo et } \\
\text { al., 2016) and used the maize U6 snRNA promoters } \\
\text { to edit the (PSY1). PSY1 is active in the biosynthetic } \\
\text { pathway of carotenoids, and its allele (psy1) causes } \\
\text { white seeds or albino seeds. } 7 \text { M0 lines were } \\
\text { confirmed to bear the psy1 cutoff trait between fifty }\end{array}$} & $\begin{array}{l}\text { two M0 benefits by Agrobacterium-mediated } \\
\text { mutation, but all } 7 \text { lines were deep transcribed to } \\
\text { recognize the requirement of variance and to assess } \\
\text { the mutation quality. No in sites were modified, and } \\
\text { healthy psy1 variants were collected (Feng et al. } \\
\text { (2016) and have demonstrated the efficacy of the } \\
\text { CRISPR/Cas9 method in maize by accessing the } \\
\text { albino marker-gene using an explants system. The sg- } \\
\text { RNA was engineered to reach a region in the eight } \\
\text { exons of Zmzb7 and the maize U3 promoters had } \\
\text { been used for expression, resulting in albino plants. } \\
\text { Two lines have been shown to have } 31 \% \text { mutation } \\
\text { efficiency after Agrobacterium-mediated }\end{array}$ \\
\hline
\end{tabular}

[Citation: Farooq, M.U., Bashir, M.F., Khan, M.U.S, Iqbal, B., Ali, Q. (2021). Role of CRISPR to improve abiotic stress tolerance in crop plants. Biol. Clin. Sci. Res. J., 2021: 69. doi: https://doi.org/10.54112/bcsrj.v2021i1.69] 
transformation of maize embryo. Other than model plants, the CRISPR/Cas9 genetic modification technique has been used to improve important traits in many other monocot crops (Kapusi et al., 2017). The (ENGase) gene was knocked out during barley using CRISPR/Cas9. ENGase was knocked out using particles disruption or Agrobacterium-mediated conversion with a set of 5 gRNAs. The mutational output of T0 to T1 mutant barley line was found to be $78 \%$. In functional genes, such knockout crops would be important for analyzing gene expression (Kaur $e t$ al., 2018). CRISPR/Cas9 modification was shown in banana cultivars for RAS-PDS gene, which plays a significant role in the biosynthesis of carotenoids. The use of CRISPR to knock out RAS-PDS in bananas resulted in thirteen mutants that were tested for carotenoid \& chlorophyll content.

\section{Dicots (Arabidopsis)}

Arabidopsis to show recombinant target genetic modifications for the very first time 3 Arabidopsis genes linked to phenology (Feng et al. (2013). The genes for (BRI1) AND (JAZ1), \& (GAI) were edited then genotyped using the floral dip process. Further sampling validated the genetic recombination efficiency (26-84\%) (Pan et al., 2016) and used CRISPR/Cas9 genetic modifications in Arabidopsis to modify albinism-related genomes, (CHLI1) \& CHLI2, with mutated plants screened using Increased Fragment Length-Polymorphism. They showed how crucial the modern genome editing technology is for gene correction and elimination of huge genomic segments in plant genomes. CRISPR/Cas9 genetic modification was used to investigate the effectiveness, heredity, accuracy, and sequence of transformed genes (Feng et al., 2014)

\section{Cotton}

Cotton seeds have substantial oil stocks, making it a good choice for biofuels in comparison to becoming a fiber crop (Fang et al., 2016; Ji et al., 2015). The gene encoding of $G$. hirsutum has been published ( $\mathrm{Li}$ et al., 2015). CRISPR tools can now be used to make accurate DNA changes (Janga et al., 2017). The CRISPR/Cas9 method was used to perform selective genetic modification in cotton for the first time. Three reference sites throughout the GFP sequence were used as a keeping quality for activatory in green fluorescent protein (GFP) integrated in genetically modified cotton. Seven of around nine M0 plantlets tested for gRNA2 knockouts showed homozygosis changes, while another nine demonstrated biallelic indels markers (Hayut et al., 2017; Janga et al., 2017).

\section{Soybean}

Soybean other name (Glycine max L.) is a major seed oil plant with a higher protein content in the seeds.
The seeds also include a number of biologically active compounds (Cai et al., 2015). To effectively accomplish recombinant genetic modifications in soybean, the researchers used a simple sgRNA for a gene of interest (bar) or six sgRNAs that targeted separate sites of two nominal soybean genes (GmFEI2 \& GmSHR), and then tested the effectiveness of the sgRNAs in such hairy plant roots. Small deletions and insertions resulted from mutagenesis of specific gene locations in soybean chromosome four (DD20 or DD43) (Li et al., 2015). At the callus point, specific PCR analysis identified targeted gene compatibility by HDR. In comparison to Arabidopsis AtU6 to 26 promoters, the soybean GmU6-16-1 promoters was thought to be highly effective in simultaneously editing several homoeoalleles (Du et al., 2016).

\section{Tomato}

Because of availability of effective transformation methods, practical genomic analysis, and extensive history on quality assurance, tomato (Solanum lycopersicum L.) is an excellent candidate for studying CRISPR/Cas9 genetic modification (Pan et al., 2016: Brooks et al., 2014; Tang et al., 2016). SlAGO7, the tomato ARGONAUTE gene, were discovered to have effective CMGE that can be easily distinguished phenotypically because mutants formed first leaflet without petioles. The SHORT-ROOT gene was knocked out of tomatoes' hairy roots using CRISPR, indicating that gene activity is conserved between Arabidopsis \& tomato. SHR also controls transcription of the transcriptional gene (SCR) as well as root length (Ron et al., 2014). One of most important concepts in the field of fleshy fruits is ripening regulation.

\section{Potato}

Potato is a staple food for global food production, although with global warming, it's critical to breed potatoes to adapt and also find breeding materials that could be used to expand the area where they are grown. Through using CMGE to mutate the (GBSS) gene in potato, the waxy genetic diversity was established. Classification of starch in genetic code lines showed only the existence of amylopectin and no amylose, suggesting that all four (GBSS) alleles were knocked out (Andersson et al., 2017). ACETOLACTATE SYNTHASE one has also been mutated in potatoes to achieve multiallelic mutants StALS1 (Bertier et al., 2018; Butler et al., 2016).

\section{Conclusion}

Selective breeding technologies require scientists to inject desired traits more accurately and rapidly than traditional breeding. CRISPR/Cas9-based genetic modification is a great technology. In the future, crops enhancement using genome editing techniques

[Citation: Farooq, M.U., Bashir, M.F., Khan, M.U.S, Iqbal, B., Ali, Q. (2021). Role of CRISPR to improve abiotic stress tolerance in crop plants. Biol. Clin. Sci. Res. J., 2021: 69. doi: https://doi.org/10.54112/bcsrj.v2021i1.69] 
to increase yield, nutrient content, disease tolerance, as well as other traits would be a major focus. It has been used extensively in many different plants for practical research, countering biotic \& abiotic stresses, and improving other essential agronomic ally important traits in the last five years. While some changes to this technology are needed to increase ontarget performance, the majority of the work done so far is tentative and needs improvement. Nonetheless, CRISPR/Cas9-based genetic engineering will grow in popularity or become a necessary technique for obtaining 'suitably edited' crops that will aid in achieving the zero hunger target and feeding the world's growing population.

\section{Conflict of interest}

The authors declared absence of conflict of interest.

\section{References}

Andersson, M., Turesson, H., Nicolia, A., Falt, A. S., Samuelsson, M., and Hofvander, P. (2017). Efficient targeted multiallelic mutagenesis in tetraploid potato (Solanum tuberosum) by transient CRISPR-Cas9 expression in protoplasts. Plant Cell Reports 36, 117-128.

Bertier, L. D., Ron, M., Huo, H., Bradford, K. J., Britt, A. B., and Michelmore,R. W. (2018). High-resolution analysis of the efficiency, heritability, andediting outcomes of CRISPRCas9 -induced modifications of NCED4 in lettuce (Lactuca sativa). G3 8: 1513-1521.

Brooks, C., Nekrasov, V., Lippman, Z. B., and Van Eck, J. (2014). Efficient gene editing in tomato in the first generation using the clustered regularly interspaced short palindromic repeats/CRISPR-associated9 system. Plant Physiology 166, 1292-1297.

Butler, N. M., Baltes, N. J., Voytas, D. F., and Douches, D. S. (2016). Geminivirus-mediated genome editing in potato (Solanum tuberosum L.) using sequence- specific nucleases. Frontiers in Plant Science 7:1045.01045

Cai, Y., Chen, L., Liu, X., Guo, C., Sun, S., Wu, C., et al. (2018). CRISPR/Cas9-mediated targeted mutagenesis of GmFT2a delays flowering time in soybean. Plant Biotechnology Journal 16, $176-185$.

Cermak, T., Doyle, E. L., Christian, M., Wang, L., Zhang, Y., Schmidt, C., et al.(2011). Efficient design and assembly of custom TALEN and other TAL effectorbased constructs for DNA targeting. Nucleic Acids Research 39:e82.

Chen, K., and GAO, C. (2013). TALENs: customizable molecular DNA scissors for

Du, H., Zeng, X., Zhao, M., Cui, X., Wang, Q., Yang, H., et al. (2016). Efficienttargeted mutagenesis in soybean by TALENs and
CRISPR/Cas9. Journal of Biotechnology 217, 90-97.

Endo, A., Masafumi, M., Kaya, H., and Toki, S. (2016). Efficient targeted mutagenesis of rice and tobacco genomes using Cpf1 from Francisella novicida. Scientific Reports 6:38169.

Fang, Y., and Tyler, B. M. (2016). Efficient disruption and replacement of aneffector gene in the Oomycete Phytophthora sojae using CRISPR/Cas9. Molecular Plant Pathology 17, 127-139.

Feng, C., Yuan, J., Wang, R., Liu, Y., Birchler, J. A., and Han, F. (2016). Efficienttargeted genome modification in maize using CRISPR/Cas9 system. Journal of Genetics and Genomics 43, 37-43.

Feng, Z., Mao, Y., Xu, N., Zhang, B., Wei, P., Yang, D. L., et al. (2014).Multigeneration analysis reveals the inheritance, specificity, and patterns of CRISPR/Cas-induced gene modifications in Arabidopsis. Proceedings National Academy Science USA 111, 4632-4637.

Gaj, T., Gersbach, C. A., and Barbas, C. F. III (2013). ZFN, TALEN, and CRISPR/Cas-based methods for genome engineering. Trends in Biotechnology 31,397-405.

Gil-Humanes, J., Wang, Y., Liang, Z., Shan, Q., Ozuna, C. V., Sanchez-Leon, S., et al. (2017). High-efficiency gene targeting in hexaploid wheat using DNA replicons and CRISPR/Cas9. Plant Journal 89, 1251-1262.

Govindan, G., and Ramalingam, S. (2016). Programmable site-specific nucleasesfor targeted genome engineering in higher eukaryotes. Journal of Cell Physiology 231,2380-2392.

Hayut, S. F., Melamed Bessudo, C., and Levy, A. A. (2017). Targeted recombination between homologous chromosomes for precise breeding in tomato. Nature Communication 8:15605.

Hu, X., Meng, X., Liu, Q., Li, J., and Wang, K. (2018). Increasing the efficiencyof CRISPRCas9-VQR precise genome editing in rice. Plant Biotechnology Journal 16,292-297.

Ishino, Y., Shinagawa, H., Makino, K., Amemura, M., and Nakata, A. (1987).Nucleotide sequence of the IAP gene, responsible for alkaline phosphataseisozyme conversion in Escherichia coli, and identification of the gene product. Journal of Bacteriology 169, 5429-5433.

Ito, Y., Nishizawa-Yokoi, A., Endo, M., Mikami, M., and Toki, S. (2015).CRISPR/Cas9-mediated mutagenesis of the RIN locus that regulates tomato fruit ripening. Biochemical and 
Biophysical Research Communications 467, 76-82.

Janga, M. R., Campbell, L. M., and Rathore, K. S. (2017). CRISPR/Cas9-mediatedtargeted mutagenesis in upland cotton (Gossypium hirsutum L.). Plant Molecular Biology 94, 349360.

Jansen, R., Embden, J. D. V., Gaastra, W., and Schouls, L. M. (2002). Identification of genes that are associated with DNA repeats in prokaryotes. Molecular Microbiology 43, 15651575.

Ji, X., Zhang, H., Zhang, Y., Wang, Y., and Gao, C. (2015). Establishing a CRISPRCas-like immune system conferring DNA virus resistance in plants. Nature Plants 1:15144.

Jiang, W., Zhou, H., Bi, H., Fromm, M., Yang, B., and Weeks, D. P. (2013).Demonstration of CRISPR/Cas9/sgRNA-mediated targeted gene modificationin Arabidopsis, tobacco, sorghum and rice. Nucleic Acids Research 41:e188.

Jinek, M., Chylinski, K., Fonfara, I., Hauer, M., Doudna, J. A., and Charpentier,(2012). A programmable dual-RNA-guided DNA endonuclease in adaptivebacterial immunity. Science 337, 816-821.

Kapusi, E., Corcuera-Gomez, M., Melnik, S., and Stoger, E. (2017). Heritablegenomic fragment deletions and small indels in the putative engase geneinduced by CRISPR/Cas9 in barley. Frontiers in Plant Science 8:540.

Karkute, S. G.,Singh, A. K., Gupta, O. P., Singh, P. M., and Singh, B. (2017).CRISPR/Cas9 mediated genome engineering for improvement of horticulturalcrops. Frontiers in Plant Science 8: 1635 .

Kaur, N., Alok, A., Shivani Kaur, N., Pandey, P., Awasthi, P., and Tiwari, S.(2018). CRISPR/Cas9-mediated efficient editing in phytoene desaturase (PDS) demonstrates precise manipulation in banana cv. Rasthali genome. Functional and Integrated Genomics 18, 89-99.

Kim, D., Alptekin, B., and Budak, H. (2018). CRISPR/Cas9 genome editing in wheat. Funct. Integr. Genomics 18, 31-41.

Lee, C. M., Cradick, T. J., and Bao, G. (2016). The Neisseria meningitidis CRISPRCas9 system enables specific genome editing in mammalian cells. Molecular Therapy 24, 645-654.

Li, C., Hao, M., Wang, W., Wang, H., Chen, F., Chu, W., et al. (2018). An efficient CRISPR/cas9 platform for rapidly generating simultaneous mutagenesis of multiple gene homoeologs in allotetraploid oilseed rape. Frontiers in Plant Science 9:442.

Li, C., Unver, T., and Zhang, B. (2017). A highefficiency CRISPR/Cas9 systemfor targeted mutagenesis in Cotton (Gossypium hirsutum L.). Science Reports 7:43902.

Li, F., Fan, G., Lu, C., Xiao, G., Zou, C., Kohel, R. J., et al. (2015). Genome sequence of cultivated upland cotton (Gossypium hirsutum TM-1) provides insights into genome evolution. Nature Biotechnology 33, 524-530.

Ma, X., Zhu, Q., Chen, Y., and Liu, Y. G. (2016). CRISPR/Cas9 platformsfor genome editing in plants: developments and applications. Molecular Plant 9,961-974.

Mali, P., Aach, J., Stranges, P. B., Esvelt, K. M., Moosburner, M., Kosuri, S., et al. (2013). CAS9 transcriptional activators for target specificity screeningand paired nickases for cooperative genome engineering. Nature Biotechnology 31, 833-838.

Malnoy, M., Viola, R., Jung, M. H., Koo, O. J., Kim, S., Kim, J. S., et al. (2016). DNAFree genetically edited grapevine and apple protoplast using CRISPR/Cas9 ribonucleoproteins. Frontiers in Plant Science 7:1904.

Osakabe, Y., and Osakabe, K. (2015). Genome editing with engineered nucleases inplants. Plant Cell Physiology 56, 389-400.

Osakabe, Y., Watanabe, T., Sugano, S. S., Ueta, R., Ishihara, R., Shinozaki, K., et al.(2016). Optimization of CRISPR/Cas9 genome editing to modify abiotic stress responses in plants. Science Reports 6:26685.

Pan, C., Ye, L., Qin, L., Liu, X., He, Y., Wang, J., et al. (2016). CRISPR/Cas9-mediated efficient and heritable targeted mutagenesis in tomato plants in thefirst and later generations. Science Reports 6:24765.

Pauwels, K., Podevin, N., Breyer, D., Carroll, D., and Herman, P. (2014).Engineering nucleases for gene targeting: safety and regulatory considerations. Nature Biotechnology 31, 1827.

Ran, F. A., Cong, L., Yan, W. X., Scott, D. A., Gootenberg, J. S., Kriz, A. J., et al.(2015). In vivo genome editing using Staphylococcus aureus Cas9. Nature 520,186-191.

Ren, C., Liu, X., Zhang, Z., Wang, Y., Duan, W., Li, S., et al. (2016). CRISPR/Cas9mediated efficient targeted mutagenesis in chardonnay (Vitis vinifera L.). Science Reports 6:32289.

Ricroch, A., Clairand, P., and Harwood, W. (2017). Use of CRISPR systems in plant genome

[Citation: Farooq, M.U., Bashir, M.F., Khan, M.U.S, Iqbal, B., Ali, Q. (2021). Role of CRISPR to improve abiotic stress tolerance in crop plants. Biol. Clin. Sci. Res. J., 2021: 69. doi: https://doi.org/10.54112/bcsrj.v2021i1.69] 
editing: toward new opportunities in agriculture. Emerg. Top. Life Sci.1, 169-182.

Ron, M., Kajala, K., Pauluzzi, G., Wang, D., Reynoso, M. A., Zumstein, K., et al. (2014). Hairy root transformation using Agrobacterium rhizogenes asa tool for exploring cell typespecific gene expression and function using tomato as a model. Plant Physiology 166, 455469.

Shan, Q., Wang, Y., Li, J., and GAO, C. (2014). Genome editing in rice and wheat using the CRISPR/Cas system. Nature Protocol 9, 23952410.

Shan, Q., Wang, Y., Li, J., Zhang, Y., Chen, K., Liang, Z., et al. (2013).Targeted genome modification of crop plants using a CRISPRCas system. Nature Biotechnology 31, 686-688.

Shi, J., GAO, H., Wang, H., Lafitte, H. R., Archibald, R. L., Yang, M., et al. (2017).ARGOS8 variants generated by CRISPR-Cas9 improve maize grain yield under Field drought stress conditions. Plant Biotechnology Journal 15, 207-216.

Shimatani, Z., Kashojiya, S., Takayama, M., Terada, R., Arazoe, T., Ishii, H., et al. (2017). Targeted base editing in rice and tomato using a CRISPR-Cas9 cytidine deaminase fusion. Nature Biotechnology 35, 441-443.

Tang, F., Yang, S., Liu, J., and Zhu, H. (2016). Rj4, a gene controlling nodulationspecificity in soybeans, encodes a thaumatin-like protein but not the onepreviously reported. Plant Physiology 170, 26-32.

Ueta, R., Abe, C., Watanabe, T., Sugano, S. S., Ishihara, R., Ezura, H., et al. (2017).Rapid breeding of parthenocarpic tomato plants using CRISPR/Cas9. Science Reports 7:507.

Waltz, E. (2018). With a free pass, CRISPR-edited plants reach market in record time. Nature Biotechnology 36, 6-7.

Wang, F., Wang, C., Liu, P., Lei, C., Hao, W., Gao, Y., et al. (2016). Enhanced riceblast resistance by CRISPR/Cas9-targeted mutagenesis of the ERF transcription factor gene OsERF922. PLoS One 11: 0154027.

Wang, Z. P., Xing, H. L., Dong, L., Zhang, H. Y., Han, C. Y., Wang, X. C.,et al. (2015). Egg cellspecific promoter-controlled CRISPR/Cas9 efficientlyenerates homozygous mutants for multiple target genes in Arabidopsis in asingle generation. Genome Biology 16:144.

Watanabe, K., Oda-Yamamizo, C., Sage-Ono, K., Ohmiya, A., and Ono, M. (2018).Alteration of flower colour in Ipomoea nil through CRISPR/Cas9-mediated mutagenesis of carotenoid cleavage dioxygenase 4. Transgenic Research 27, 25-38.

Xie, K., and Yang, Y. (2013). RNA-guided genome editing in plants using aCRISPR-Cas system. Molecular Plant 6, 1975-1983.

Xu, H., Xiao, T., Chen, C. H., Li, W., Meyer, C. A., $\mathrm{Wu}, \mathrm{Q}$., et al. (2015). Sequencedeterminants of improved CRISPR sgRNA design. Genome Research 25, 1147-1157.

Yang, Y., Zhu, G., Li, R., Yan, S., Fu, D., Zhu, B., et al. (2017). The RNA editing factor SIORRM4 is required for normal fruit ripening in tomato. Plant Physiology 175, 1690-1702.

Zetsche, B., Heidenreich, M., Mohanraju, P., Fedorova, I., Kneppers, J., DeGennaro, E. M., et al. (2017). Multiplex gene editing by CRISPR-Cpf1 usinga single crRNA array. Nature Biotechnology 35, 31-34.

Zhang, F., LeBlanc, C., Irish, V. F., and Jacob, Y. (2017). Rapid and efficient CRISPR/Cas9 gene editing in citrus using the YAO promoter. Plant Cell Reports 36, 1883-1887.

Zhang, H., Zhang, J., Wei, P., Zhang, B., Gou, F., Feng, Z., et al. (2014). TheCRISPR/Cas9 system produces specific and homozygous targeted gene editing in rice in one generation. Plant Biotechnology Journal. 12, 797-807.

Zhang, Z., Ge, X., Luo, X., Wang, P., Fan, Q., Hu, G., et al. (2018). Simultaneous editing of two copies of Gh14-3-3d confers enhanced transgene-clean plant defense against Verticillium dahliae in allotetraploid upland cotton. Frontiers in Plant Science 7: 842

Zhang, Y., Zhang, F., Li, X., Baller, J. A., Qi, Y., Starker, C. G., et al. (2013). Transcription activator-like effector nucleases enable efficient plant genome engineering. Plant Physiology 161, 20-27.

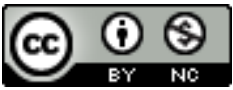

Open Access This article is licensed under a Creative Commons Attribution 4.0 International License, which permits use, sharing, adaptation, distribution and reproduction in any medium or format, as long as you give appropriate credit to the original author(s) and the source, provide a link to the Creative Commons licence, and indicate if changes were made. The images or other third party material in this article are included in the article's Creative Commons licence, unless indicated otherwise in a credit line to the material. If material is not included in the article's Creative Commons licence and your intended use is not permitted by statutory regulation or exceeds the permitted use, you will need to obtain permission directly from the copyright holder. To view a copy of this licence, visit http://creativecommons.org/licen ses/by/4.0/. (C) The Author(s) 2021

[Citation: Farooq, M.U., Bashir, M.F., Khan, M.U.S, Iqbal, B., Ali, Q. (2021). Role of CRISPR to improve abiotic stress tolerance in crop plants. Biol. Clin. Sci. Res. J., 2021: 69. doi: https://doi.org/10.54112/bcsrj.v2021i1.69] 